\title{
Radiation Damage of Fe-based Nanocrystalline Materials
}

\author{
J. Sitek*, J. Dekan, M. Pavlovič \\ Institute of Nuclear and Physical Engineering, Faculty of Electrical Engineering and Information Technology, Slovak \\ University of Technology in Bratislava, Ilkovičova 3, 81219 Bratislava, Slovakia

\begin{abstract}
Nanocrystalline $\left(\mathrm{Fe}_{1-x} \mathrm{Ni}_{x}\right)_{81} \mathrm{Nb}_{7} \mathrm{~B}_{12}(x=0,0.25,0.5,0.75)$ and Vitroperm alloys were studied by Mössbauer spectroscopy after neutron irradiation at fluences of $10^{16} \mathrm{n} / \mathrm{cm}^{2}$ and $10^{17} \mathrm{n} / \mathrm{cm}^{2}$. From structure analyses, we have identified ferromagnetic bcc-FeNi in nanocrystalline state and partly paramagnetic $(\mathrm{Fe}-\mathrm{Ni})_{23} \mathrm{~B}_{6} \mathrm{phase}$. Neutron irradiation had an influence on the magnetic microstructure, which is manifested in the change of the Mössbauer parameters, such as the direction of net magnetic moment, the intensity of internal magnetic field and the volumetric fraction. The results indicate that the changes of the microscopic magnetic parameters, induced by neutron irradiation, depend on iron and nickel content. Significant radiation damage started at neutron fluence of $10^{17} \mathrm{n} / \mathrm{cm}^{2}$.
\end{abstract}

DOI: $10.12693 /$ APhysPolA.126.84

PACS: $33.45 .+\mathrm{x}, 61.46 .+\mathrm{w}, 75.50 . \mathrm{Kj}$

\section{Introduction}

In the recent years, nanocrystalline alloys have become attractive for applications. The most prominent FINEMET, NANOPERM and HITPERM have been frequently investigated because they exhibit excellent soft magnetic properties. It was also shown [1] that some physical properties of nanocrystalline materials are affected by neutron irradiation. Changes in the orientation of the average magnetic moment were observed in neutron irradiated metallic glasses and nanocrystals [2]. The particle bombardment produces defects that may cause realignment of magnetic domains, implying reorientation of magnetic moments. The change in the local neighbourhoods of atoms affects the average hyperfine magnetic field as well as the shape of hyperfine field distributions.

In the case of nanocrystalline alloys consisting of crystalline nanograins embedded in an amorphous intergranular matrix, the irradiation by neutrons leads to redistribution of atoms in the amorphous matrix, disturbance of regular atomic ordering of the crystal lattice and atom exchange between the amorphous and crystalline component. The mechanism of the radiation damage also depends on the constituent elements. Each of them possesses different cross-section to thermal and fast neutrons.

Using Mössbauer spectroscopy, information can be gained on changes in the orientation of the net magnetic moment, in the magnitude of the magnetic hyperfine field as well as the volumetric fraction of the crystalline and the amorphous component after irradiation.

Our work is focused on the structural and local magnetic modification induced in selected alloys by neutron irradiation at different fluences.

*corresponding author; e-mail: jozef.sitek@stuba.sk

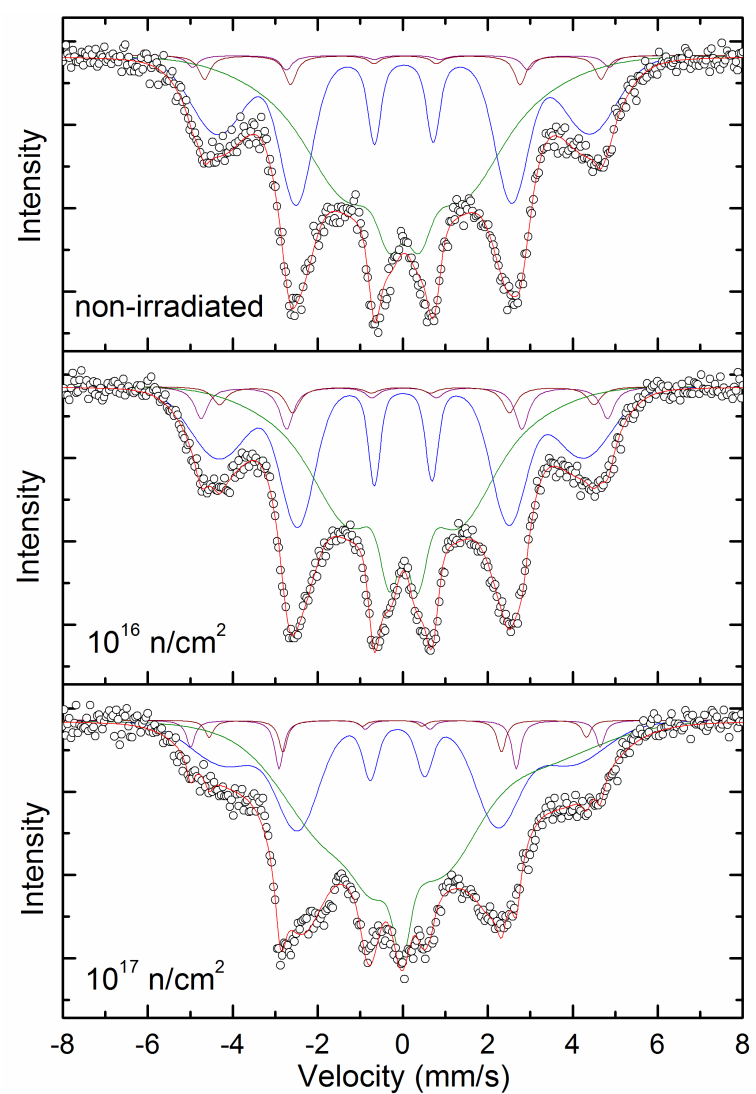

Fig. 1. Mössbauer spectra of nanocrystalline $\left(\mathrm{Fe}_{1-x} \mathrm{Ni}_{x}\right)_{81} \mathrm{Nb}_{7} \mathrm{~B}_{12}$ for $x=0.5$.

\section{Experimental details}

Ribbon-like specimens of the master alloy were prepared by planar flow casting. The ribbons were about $25 \mu \mathrm{m}$ thick and $10 \mathrm{~mm}$ wide. The nominal composition was $\left(\mathrm{Fe}_{1-x} \mathrm{Ni}_{x}\right)_{81} \mathrm{Nb}_{7} \mathrm{~B}_{12}$ (where $x=0,0.25$, $0.5,0.75)$ and $\mathrm{Fe}_{73} \mathrm{Cu}_{1} \mathrm{Nb}_{3} \mathrm{Si}_{16} \mathrm{~B}_{7}$ (Vitroperm). Annealing was carried out in vacuum at the temperature of 
$550{ }^{\circ} \mathrm{C}$ for 1 hour. Samples were irradiated in a nuclear reactor at fluences of $10^{16} \mathrm{n} / \mathrm{cm}^{2}$ and $10^{17} \mathrm{n} / \mathrm{cm}^{2}$. Mössbauer spectra were collected in transmission geometry by a conventional constant-acceleration spectrometer with a ${ }^{57} \mathrm{Co}(\mathrm{Rh})$ source. All spectra were measured at room temperature. The spectra were evaluated by the CONFIT program [3], which allows simultaneous treatment of crystalline and residual amorphous phase by means of individual lines and distribution of hyperfine components.

TABLE I

Parameters of Mössbauer spectra of the $\left(\mathrm{Fe}_{1-x} \mathrm{Ni}_{x}\right)_{81} \mathrm{Nb}_{7} \mathrm{~B}_{12}$ and Vitroperm alloys; amorphous component: $A_{23 m}$ parameter, $B_{a m}$ - average value of magnetic induction of internal magnetic field, $A_{a m}$ - volumetric fraction; crystalline component: $A_{23 c r}$ parameter, $B_{c r}$ - average value of magnetic induction of internal magnetic field, $A_{c r}$ - volumetric fraction. For each material, data in the $1^{\text {st }}$, the $2^{\text {nd }}$ and the $3^{\text {rd }}$ row corresponds to the fluence of $0 \mathrm{n} / \mathrm{cm}^{2}$ (non-irradiated), $10^{16} \mathrm{n} / \mathrm{cm}^{2}$ and $10^{17} \mathrm{n} / \mathrm{cm}^{2}$, respectively.

\begin{tabular}{c|c|c|c|c|c|c}
\hline \hline Sample & $A_{23 m}$ & $B_{a m}(\mathrm{~T})$ & $A_{a m}[\%]$ & $A_{23 c r}$ & $B_{c r}(\mathrm{~T})$ & $A_{c r}[\%]$ \\
\hline \multirow{4}{*}{$x=0$} & 1.82 & 8.19 & 84 & 1.82 & 32.86 & 16 \\
& 4.00 & 8.03 & 81 & 3.82 & 30.97 & 19 \\
& 2.23 & 7.74 & 86 & 2.24 & 32.53 & 14 \\
\hline \multirow{4}{*}{$x=0.25$} & 3.37 & 22.04 & 84 & 3.37 & 33.51 & 16 \\
& 2.89 & 23.08 & 90 & 3.25 & 33.57 & 10 \\
& 3.04 & 23.64 & 94 & 3.06 & 33.67 & 6 \\
\hline & 1.28 & 19.98 & 94 & 4.00 & 30.22 & 6 \\
$x=0.50$ & 3.60 & 19.50 & 89 & 4.00 & 28.53 & 11 \\
& 2.72 & 19.10 & 93 & 4.00 & 28.75 & 7 \\
\hline \multirow{3}{*}{$x=0.75$} & 1.66 & 25.35 & 64 & 4.00 & 28.16 & 36 \\
& 2.49 & 24.34 & 42 & 2.60 & 27.98 & 58 \\
& 1.95 & 23.75 & 45 & 3.62 & 27.97 & 55 \\
\hline \multirow{2}{*}{ Vitro- } & 2.15 & 21.04 & & & & \\
perm & 1.98 & 21.12 & & & & \\
& 1.89 & 21.25 & & & &
\end{tabular}

\section{Results and discussion}

Mössbauer spectra and parameters of non-irradiated nanocrystalline samples and samples irradiated at different fluences of neutrons $\left(10^{16} \mathrm{n} / \mathrm{cm}^{2}\right.$ and $\left.10^{17} \mathrm{n} / \mathrm{cm}^{2}\right)$, taken at room temperature are shown in Fig. 1 and in Table 1. After irradiation, the changes in the orientation of net magnetic moment, changes in the magnitude of the average hyperfine magnetic field of amorphous and crystalline components and changes in volumetric fraction take place. Orientation of net magnetic moment is reflected in relative intensity of the second and the fifth line of Mössbauer spectrum $\left(A_{23}\right)$. This parameter achieves a maximum value if the net magnetic moment lays along the sample surface and a minimum value if it is perpendicular to the sample surface. It is known that magnetic domain structure as well as magnetization processes are governed by both, the microstructural defects and their stress fields. Consequently, the results suggest that the short range order and the distribution of magnetic dipoles are affected by stresses in the irradiated regions [4]. Slight changes in the average hyperfine field and in the volumetric fraction of the amorphous component can indicate some movement of Fe atoms from the amorphous matrix to the crystalline part, contributing to slight decrease of the internal magnetic field after irradiation. We suppose that free Fe atoms originate either from the neutron capture by boron or from recoil atoms displacement. We cannot exclude that part of crystalline iron gets damaged, it means that it contains vacancies and interstitial atoms. The fluence of $10^{17} \mathrm{n} / \mathrm{cm}^{2}$ partly amorphizes the crystalline iron grains. As an evidence of this, there is a relative increase of volumetric fraction of amorphous component and slight increase of the average value of internal magnetic field after irradiation. We found by comparison of the nanocrystalline samples with their amorphous precursors, that behavior of amorphous samples was very similar to the behaviors of amorphous component in the nanocrystalline samples. We have also analyzed the Vitroperm alloy that is used in particle accelerators. The results showed similar changes in Mössbauer spectra after irradiation, as in the former cases.

\section{Conclusions}

After summarizing all obtained results, the fluence of $10^{16} \mathrm{n} / \mathrm{cm}^{2}$ is not enough to significantly damage the amorphous and crystalline structure. This fluence modifies the structure rather than damages it. This modification is manifested by change of the net magnetic moment orientation and, at higher fluences, by changes of internal magnetic field. On macroscopic level, the decrease of magnetic susceptibility has been observed. At $10^{17} \mathrm{n} / \mathrm{cm}^{2}$, some structural damage started being visible. The results indicate that these types of amorphous and nanocrystalline alloys are not damaged by neutron irradiation up to the fluence of $10^{16} \mathrm{n} / \mathrm{cm}^{2}$.

The samples have not been investigated by complementary techniques like TEM (Transmission Electron Microscopy) or XRD (X-ray Diffraction) yet. However, future experiments are planned with these techniques included. Investigations of similar materials using synchrotron radiation are currently in progress.

\section{Acknowledgments}

This work was supported by the Ministry of Education, Science, Research and Sport of the Slovak Republic via the projects VEGA 1/0286/12 and VEGA 1/1163/12.

\section{References}

[1] F.P. Shimansky, R. Gerling, R. Wagner, Mat. Sci. Eng. 97, 173 (1997).

[2] J. Degmová, Ph.D. Thesis, Slovak University of Technology, Bratislava 2001.

[3] T. Zak, Y. Jiraskova, Surf. Interface Anal. 38, 710 (2006).

[4] J. Degmová, J. Sitek, J.M. Greneche, in Properties and Applications of Nanocrystalline Alloys from Amorphous Precursors, Eds. B. Idzikowski, P. Svec, M. Miglierini, Kluwer Academic Publishers, Dodrecht 2005 . 